\title{
O perfil psicossocial de pessoas vivendo com HIV/AIDS em uma unidade de acompanhamento em Belém-PA: Relato de Experiência
}

\author{
The psychosocial profile of people living with HIV / AIDS in a follow-up unit in Belém-PA: \\ Experience Report
}
El perfil psicosocial de las personas que viven con VIH / SIDA en una unidad de seguimiento en Belém-PA: Informe de Experiencia

\begin{abstract}
Wesley Brandão Dias ${ }^{1 *}$, Chrisla Brena Malheiro Lima1, Filipe Rabelo Rodrigues ${ }^{1}$, Maria Eduarda de Oliveira Cardosoํㅜㄹ Jéssica Maria Lins da Silva ${ }^{1}$, Lorrane Teixeira Araújo ${ }^{1}$, Ana Júlia da Costa Monteiro ${ }^{1}$, Lucas Miléo Teixeira ${ }^{1}$, Victória Lima Mendes Leite ${ }^{1}$, Eliana Soares Coutinho ${ }^{1}$, Thanaira Aicha Fernandes Maciel ${ }^{1}$, José Augusto Carvalho de Araújo ${ }^{2}$, Ana Caroline Guedes Souza Martins ${ }^{1}$, Aluísio Ferreira Celestino Júnior ${ }^{3}$, Margarete Feio Boulhosa ${ }^{4}$.
\end{abstract}

\section{RESUMO}

Objetivo: Descrever a vivência de acadêmicos durante uma atuação em um campo psicossocial, expondo observações e intervenções realizadas junto a usuários com HIV/AIDS. Relato da Experiência: Estudo qualitativo do tipo relato de experiência desenvolvido através das etapas do Arco de Maguerez em uma unidade de saúde em de Belém no Pará. Desenvolveu-se uma ação com usuários de um serviço de apoio e tratamento a pessoas com HIV/AIDS, com foco na orientação, auxílio psicossocial e emocional, com atividades lúdicas. A partir das etapas constatou-se que os usuários que participaram da pesquisa encontravam-se afetados por sentimentos impostos por estigmas sociais, gerando conflitos nas relações interpessoais. Após a ação notou-se a melhora pontual do diálogo e a promoção de sentimentos de autonomia, aceitação, empoderamento e incentivo mútuo. Considerações Finais: Compreende-se que o objetivo foi alcançado e a contribuição dos profissionais de enfermagem foi de suma importância no processo de fortalecimento social e na construção de competências e habilidades necessárias ao atendimento integral e humanizado dos indivíduos.

Palavras-Chave: Soropositividade para HIV, Adaptação psicológica, Assistência integral à saúde.

\begin{abstract}
Objective: To describe the experience of academics while working in a psychosocial field, exposing observations and interventions with users with HIV / AIDS. Experience Report: A qualitative study of the type of experience report developed through the steps of the Maguerez Arch in a health unit in Belém, Pará. An action was developed with users of a support and treatment service for people with HIV / AIDS, focusing on orientation, psychosocial and emotional help, with playful activities. From the stages it was found that the users who participated in the research were affected by feelings imposed by social stigmas, generating conflicts in interpersonal relationships. After the action there was a punctual improvement of dialogue and the promotion of feelings of autonomy, acceptance, empowerment and mutual encouragement. Final Considerations: It is
\end{abstract}

1 Universidade do Estado do Pará (UEPA), Belém-PA. *E-mail: wesleybrandao437@gmail.com

2 Universidade Federal de São Carlos (UFSCAR), São Carlos-SP.

3 Universidade Federal do Pará (UFPA), Belém-PA.

4 Universidad Internacional Três Fronteras (UNINTER), Paraguai.

SUBMETIDO EM: $8 / 2019$

ACEITO EM: 9/2019

PUBLICADO EM: 1/2020

REAS/EJCH | Vol.12(1) | e1429 | DOI: https://doi.org/10.25248/reas.e1429.2020 Página 1 de 6 
understood that the objective was achieved, and the contribution of nursing professionals was of paramount importance in the process of social empowerment and in the construction of competences and skills necessary for the integral and humanized care of individuals.

Key words: HIV seropositivity, Psychological adaptation, Comprehensive health care.

\section{RESUMEN}

Objetivo: Describir la experiencia de los académicos mientras trabajan en un campo psicosocial, exponiendo observaciones e intervenciones con usuarios con VIH / SIDA. Informe de experiencia: un estudio cualitativo del tipo de informe de experiencia desarrollado a través de los pasos del Arco de Maguerez en una unidad de salud en Belém, Pará. Se desarrolló una acción con usuarios de un servicio de apoyo y tratamiento para personas con VIH / SIDA, centrándose en la orientación, ayuda psicosocial y emocional, con actividades lúdicas. Desde las etapas se descubrió que los usuarios que participaron en la investigación se vieron afectados por los sentimientos impuestos por los estigmas sociales, generando conflictos en las relaciones interpersonales. Después de la acción hubo una mejora puntual del diálogo y la promoción de sentimientos de autonomía, aceptación, empoderamiento y aliento mutuo. Consideraciones finales: se entiende que se logró el objetivo y la contribución de los profesionales de enfermería fue de suma importancia en el proceso de empoderamiento social y en la construcción de competencias y habilidades necesarias para la atención integral y humanizada de las personas.

Palabras-clave: Seropositividad al VIH, Adaptación psicológica, Atención integral a la salud.

\section{INTRODUÇÃO}

A Síndrome da Imunodeficiência Adquirida (AIDS) é uma doença infecto-parasitária, membro de um grupo de síndromes clínicas, causada pelo Vírus da Imunodeficiência Humana (HIV), a qual afeta as células do sistema imunológico e caracteriza-se por causar uma suscetibilidade a infecções por patógenos oportunistas (BROOKS GF, et al., 2014). De acordo com os dados epidemiológicos levantados pela secretaria de vigilância em saúde no período de 2007 a 2018, notificou-se um total de $169.932(68,6 \%)$ casos novos em homens e $77.82(31,4 \%)$ em mulheres (BRASIL, 2018).

A evolução da infecção pelo HIV divide-se em: aguda, assintomática ou latente e sintomática. A infecção aguda ocorre após a transmissão viral, que se estabelece pela transferência de fluídos corporais (sangue, sêmen, líquido vaginal e leite materno) por via sexual, parental ou vertical, os sintomas variam de um quadro gripal até uma síndrome similar à mononucleose. As principais características são a viremia elevada, a resposta imunológica intensa, a depleção de TCD4+ e o aumento de TCD8+, culminando na disseminação do HIV atingindo o sistema nervoso central e os linfonodos. Já durante a fase assintomática, os sintomas são inexistentes ou mínimos, podendo durar até 9 anos para o surgimento de novos sintomas. Por fim, a fase sintomática caracteriza-se pela imunodeficiência grave, com sintomas como: mal-estar, sudorese noturna, síndrome consumptiva atrelada a caquexia, diarreia crônica, fraqueza e febre (LAZZAROTTO AR, et al.,2010).

A partir da relevância do tema e da vivência de acadêmicos em uma unidade de saúde, percebeu-se que muitos usuários encontravam-se emocionalmente instáveis em virtude do tratamento, dos efeitos colaterais e, principalmente, pelo estigma social concebido por parcela majoritária da sociedade, fato esse que dificulta a adesão ao tratamento e, consequentemente, a melhora na saúde desses indivíduos (TAVARES NUL, et al., 2013).

O tratamento da doença ocorre através da terapia antirretroviral, que se apresenta como uma importante arma contra a evolução e os índices de mortalidade ligados ao HIV/AIDS. Dessa forma, é imprescindível seu uso de forma regular e disciplinada, contribuindo para minimizar os sinais e sintomas da doença, aumentar a qualidade e a expectativa de vida dos infectados (MORAES DCA, et al., 2014). Além da terapêutica medicamentosa, destaca-se a importância do acompanhamento da pessoa aidética com uma equipe 
multiprofissional, para identificar e compreender as diferentes necessidades deste indivíduo. Dentre esses enfatiza-se a função do acompanhamento psicológico, cuja assistência evita e/ou auxilia o manejo do sofrimento psíquico, bem como denota implicações diretas na qualidade de vida, posto que beneficia o enfrentamento da doença e as relações estabelecidas intra e interpessoalmente (RASERA EF, et al., 2007).

Corroborando o observado, Paschoal EP et al. (2014) afirma que a baixa adesão ao tratamento também está condicionada a falta de informação, visto que algumas pessoas creem que a recusa a terapia medicamentosa pode encurtar e diminuir dores futuras. Além disso, a literatura demonstra que pressões ambientais e sociais, como a perda de renda, discriminação e mudanças nos relacionamentos podem contribuir para o surgimento de problemas psicossociais após o diagnóstico (PONTES ALP e SANTOS EVC, 2007).

Assim, além das dificuldades encontradas no tratamento, os indivíduos infectados sofrem preconceitos diários, sendo, por vezes, utilizada a omissão como forma de garantir o emprego, convívio social e familiar. Muitos deles ainda acreditam que a doença é um castigo afirmando que aqueles que expõem a doença sofrem discriminações e julgamentos morais acerca de sua vida privada. Este estigma aumenta o medo e, sobretudo, o isolamento dessas pessoas, influenciando diretamente na busca e na adesão ao tratamento (GOMES AMT, et al., 2011).

Constata-se, portanto, que algumas manifestações de distúrbios psíquicos clássicos, como o transtorno de humor, ansiedade e principalmente a depressão, são frequentemente associadas a pessoas que vivem com HIV. Isso se deve ao fato de existir grande prevalência desses transtornos na população infectada pelo vírus ser maior que na população em geral (CAMARGO LA, et al., 2014).

Diante da análise desse cenário e utilizando a base metodológica tradicionalmente disseminada no curso de graduação em Enfermagem da Universidade do Estado do Pará (UEPA) - Metodologia da Problematização do Arco de Maguerez, que compreende a transformação da realidade da comunidade através de cinco etapas: observação da realidade; levantamento de pontos-chave; teorização; hipóteses de solução e aplicação prática à realidade (BERBEL NAN, 2011), realizou-se uma ação de educação em saúde voltada para o entendimento psicossocial de pessoas vivendo com HIV/AIDS no município de Belém, objetivando relatar a vivência de acadêmicos durante a atuação no campo da saúde psicossocial, expondo as observações feitas a partir da realidade retratada.

\section{RELATO DA EXPERIÊNCIA}

Este trabalho foi desenvolvido em uma unidade de saúde localizada na periferia de Belém-PA, a qual atende pessoas que vivem com o vírus HIV e recebem tratamento por meio da rede municipal de saúde após a deteç̧ão da presença do vírus realizada pelo Centro de Testagem e Aconselhamento (CTA).

Trata-se de um estudo qualitativo do tipo relato de experiência acerca da intervenção desenvolvida por acadêmicos do curso de graduação em Enfermagem da UEPA, que fitou transmitir orientações sobre o estabelecimento de relações sociais e familiares de pessoas vivendo com HIV/AIDS, a partir da realidade social observada durante as Atividades Integradas em Saúde (AIS) método pedagógico, que possibilita aos graduandos atuar diretamente e ativamente na comunidade, ampliando suas experiências nos campos de praticada saúde e estimulando o ensino-aprendizagem por meio da pesquisa e ações em saúde.

Para o desenvolvimento metodológico deste trabalho foi utilizada a Teoria da Problematização do Arco de Maguerez, que tem por finalidade levar informações, conscientização e melhorias a uma comunidade a partir da realidade inicialmente apresentada por esta. Sendo assim, a pesquisa foi realizada seguindo as etapas da medotologia supracitada, são elas: (1) Observação da Realidade; (2) Levantamento dos Pontos-chave; (3) Teorização; (4) Hipóteses de Soluções; e (5) Retorno a Realidade (BERBEL NAN, 2011).

Iniciando as etapas, realizou-se uma visita ao local para a observação da realidade a fim de encontrar possíveis situações-problemas que pudessem ofertar riscos à saúde física e/ou psicológica dos indivíduos acompanhados na unidade de saúde. A partir disso, em uma sala da instituição, ocorreram abordagens e 
questionamentos aos usuários a respeito da situação pós-diagnóstico do HIV, por meio de perguntas sobre o tratamento, o estado psicológico e as relações interpessoais envolvendo familiares e amigos, seguindo pela identificação das problemáticas constatadas.

Durante a segunda etapa (levantamento dos pontos-chave) discentes e professores da UEPA reuniramse para discutir as principais percepções referentes a visita inicial. Verificou-se, portanto, que a grande quantidade de entrevistados possuía abalo nas relações entre amigos e familiares pós-diagnóstico, resultando em isolamento, medo do abandono, ansiedade e baixa autoestima. Sendo relatado por alguns usuários a dificuldade em compartilhar a notícia da infecção com familiares devido ao preconceito por grande parte da população. A busca por fundamentação teórica ocorreu sequencialmente através do uso de plataformas digitais e bases de dados como Google Acadêmico, Scientific Electronic Library Online (SCIELO) e Banco de dados da Biblioteca Virtual em Saúde (BVS), a fim de encontrar na literatura um subsídio teórico-científico para este estudo e uma melhor compreensão do tema em questão.

Partindo para a quarta etapa do Arco de Maguerez, elaborou-se uma ação de intervenção em conjunto com docentes do curso. Definiu-se a partir desta a realização de uma atividade que priorizasse a escuta ativa, estimulando os usuários a dialogar com a equipe e entre si sobre suas emoções quanto ao tratamento, buscando ofertar a retirada de dúvidas e o incentivo ao tratamento de forma acolhedora e grupal, permitindo que os indivíduos apoiassem uns aos outros nas falas e, a partir dos recursos lúdicos disponibilizados, também através da escrita de cartões com mensagens de apoio, que ocorreu na finalização da atividade.

As últimas etapas, efetivadas pelas hipóteses de solução e retorno à realidade, foram desenvolvidas em meio a dificuldade de encontrar estratégias para dialogar com os usuários sobre as questões psicossomáticas que os envolvem. No entanto, conseguiu-se realizar as atividades com êxito, pois apesar de inicialmente retraídos, os participantes contribuíram compartilhando de forma aberta suas experiências e suas aflições provenientes do tratamento, retirando dúvidas e incentivando uns aos outros.

O retorno à realidade se deu pela execução da atividade planejada, realizada nos corredores da unidade contando com a participação de usuários que aguardavam atendimento. Após a explicação do intuito da ação, aceitação e posterior realização, confeccionou-se um cartaz com palavras de apoio formuladas pelos próprios participantes, que posteriormente foi exposto no auditório da unidade.

Por fim, todos os participantes escreveram cartões com mensagens de apoio, empoderamento e encorajamento. Posteriormente, as respostas foram unidas e expostas em um cartaz. Dentre as frases escritas nos cartões encontrava-se: "Você não está sozinho", "Respira, não pira", "Não desista", "Coragem, você é normal como qualquer outra pessoa", "Perceverança", "Determinação, persistência e amor próprio", "Nada na vida é o fim, apenas um novo recomeço. Aproveite a vida normalmente", "Nunca desista".

Através da ação concluiu-se que o objetivo principal foi alcançado, considerando que ocorreu a sensibilização dos participantes, bem como contribuiu diretamente na autoestima e no fortalecimento emocional. Além disso, considerando que pacientes nesse quadro podem desenvolver transtornos mentais em decorrência do estigma social causada pela infecção, salienta-se sobre a importância de um atendimento integralizado, que priorize as necessidades e fragilidades dos indivíduos em todos os aspectos biopsicossociais que estão inseridos.

\section{DISCUSSÃO}

Por meio do diálogo com alguns usuários da unidade de saúde, verificou-se o sentimento de solidão devido a reação de alguns familiares e amigos frente a infecção pelo HIV. Em virtude disso, constatou-se o aparecimento de síndromes psiquiátricas clássicas como o transtorno de humor, ansiedade e, particularmente, a depressão. De acordo com as pesquisas bibliográficas associadas à realidade observada na instituição, a temática abordada tem grande importância na unidade de acompanhamento, visto que 0 HIV/AIDS ainda constitui um relevante problema de saúde pública no país e pode configurar-se como um agravante para o adoecimento mental das pessoas que vivem com a infecção (MEDEIROS B, et al., 2013; BRASIL, 2018). 
Em consonância com o pensamento de Pontes ALP e Santos EVC (2007), a infecção traz diversos malefícios e incertezas para o indivíduo. Somado a isso, as relações pessoais e interpessoais como a familiar e laboral, tornam-se áreas sensíveis frente às mudanças da nova realidade acompanhada por sentimento de culpa, tristeza e rejeição. Deste modo, a preservação da qualidade de vida associada as influências sociais são fatores essenciais à manutenção da saúde física e mental, devendo os profissionais estimular e promover perspectivas positivas aos que estão sob seus cuidados (OLIVEIRA FBM, et al., 2015).

Ademais, o bom convívio entre família, amigos e a sociedade como um todo, torna-se também um fator que determina a continuidade do tratamento para a infecção, pois o apoio destes grupos pode amenizar 0 impacto das experiências estressantes vivenciadas pelos indivíduos. Posto que, pessoas que são diagnosticados com HIV e não possuem um suporte positivo dos familiares, podem encontrar-se desamparados e com maior dificuldade em continuar com o tratamento, podendo ainda desencadear a depressão e a desesperança (SILVA JB, et al., 2015). Pode-se visualizar na prática que a infecção pelo vírus HIV traz com sigo uma demanda para o indivíduo no que diz respeito ao seu autocuidado, que geralmente encontra-se fragilizado, por conta de uma nova conduta adotada pela indivíduo infectado, que se abstém desta influenciado por fatores condicionantes básicos, como aqueles referente ao seu convívio com a sociedade e família, em decorrência do grande estigma social criado por este grupo, tendo grande repercussão no seu estado de saúde deste indivíduo (NASSER CAC e NEMES MIB, 2016).

A qualidade de vida das pessoas vivendo com HIV/AIDS não se restringe apenas ao tratamento com antirretroviral, pois o seu convívio na sociedade sem fatores estressores e desencadeantes de ansiedade e transtornos de humor, também auxiliam na melhora qualidade de vida. Se o convívio social deste individuo estiver prejudicado, ele pode facilmente abandonar o tratamento e desenvolver um prognóstico sombrio causado pelo estado infeccioso. Desse modo, faz-se necessário agregar uma maior importância para os agravos sociais nestes indivíduos, bem como os fatores psicológicos que têm grande influência para a adesão ou abandono do tratamento antirretroviral e no processo de reabilitação social (CALVETTE PU, et al., 2017).

Ademais, a função do profissional na vida dessas pessoas também é um aspecto fundamental que influencia diretamente no tratamento e no enfrentamento da doença, sendo ele responsável por incentivar e auxiliar o indivíduo em todas as suas necessidades, bem como oferecer suporte contínuo e melhorar a autoimagem e autoestima das pessoas vivendo com HIV/AIDS. Evidenciou-se através de estudos que o debate acerca da função do profissional é escasso, portanto, enfatiza-se a necessidade de uma educação continuada voltada ao atendimento biopsicossocial do indivíduo, visando o equilíbrio em todas essas áreas. Desse modo, a capacitação profissional para atuar com o público em questão, de forma holística e acolhedora, faz-se primordial, o que reflete em melhorias no atendimento que consequentemente contribuirá para na qualidade de vida destas pessoas (HONORATO EJSA, et al., 2018).

Portanto, a partir das análises apresentadas salienta-se que a convivência com HIV/AIDS ainda representa um grande desafio em todas as faixas etárias, tendo como principais eixos de fragilidade o contexto biopsicossocial em que o indivíduo está inserido, bem como as dificuldades enfrentadas por eles, principalmente relacionadas ao preconceito por conta da infecção, discriminação em locais de saúde, piadas pejorativas, ataques físicos, entre outros (GOMES HN, et al., 2019). Destarte, avulta-se a necessidade da melhora na autoestima e no processo de enfrentamento da infecção causada pelo HIV, bem como uma constante capacitação de profissionais que possam identificar e compreender as necessidades decorrentes do HIV/AIDS de uma forma holística, integral, humanizada e acolhedora.

Considera-se que este trabalho contribuiu ativamente para a enfermagem, posto a importância deste profissional na orientação e no cuidado desses indivíduos. Nesse sentido, também se enfatiza o papel do acadêmico na comunidade, a fim de adquirir competências e habilidades necessárias ao desenvolvimento de sua profissão. Por fim, espera-se que esta produção fomente pesquisas futuras que visem a elaboração de estratégias para estimular a auto aceitação e promover o empoderamento e esclarecimento social quanto à temática apresentada. 


\section{CONSIDERAÇÕES FINAIS}

Este estudo buscou compreender os aspectos referentes ao perfil psicossocial de pessoas vivendo com HIV/AIDS em uma unidade de acompanhamento em Belém-PA. Por meio deste, espera-se que o trabalho subsidie e norteie futuras produções científicas nessa área. Ademais, ratifica-se a importância da desmistificação social contínua frente as pessoas vivendo com HIV/AIDS, objetivando promover uma maior integração dos aspectos que envolvem o cuidado e o bem-estar desses indivíduos.

\section{REFERÊNCIAS}

1. BERBEL NAN. As metodologias ativas e a promoção da autonomia de estudantes. Semina: Ciências Sociais e Humanas, 2011; 32(1): 25-40.

2. BRASIL. Ministério da Saúde. Secretaria de Vigilância em Saúde. HIV AIDS 2018. Boletim Epidemiológico 2018; 49(53): 01-72.

3. BROOKS GF, et al. Microbiologia Médica. Porto Alegre: AMGH, 2014; 526p.

4. CALVETTI PU, et al. Níveis de ansiedade, estresse percebido e suporte social em pessoas que vivem com HIV/Aids. Psicologia: Teoria e Pesquisa, 2016; 32 (4):01-04.

5. CAMARGO LA, et al. Saúde mental, suporte familiar e adesão ao tratamento: associações no contexto HIV/Aids. Psico USF, 2014; 19(2): 221-232.

6. GOMES AMT, et al. Representações sociais da AIDS para pessoas que vivem com HIV e suas interfaces cotidianas. Revista Latino-americana de Enfermagem, 2011; 19(3): 485-492.

7. GOMES HN, et al. Análise do atendimento nos serviços de saúde entre pessoas vivendo com HIV/AIDS. Journal of Health \& Biological Sciences, 2019; 7(4): 387-394.

8. HONORATO EJSA, et al. Percepção de profissionais de saúde sobre adesão e não adesão ao tratamento antirretroviral com pacientes portadores de HIV/AIDS. Revista Amazônica, 2018; 21(1): 116-128.

9. LAZZAROTTO AR, et al. HIV/AIDS e Treinamento Concorrente: a Revisão Sistemática. Revista Brasileira de Medicina do Esporte, 2010; 16(2): 149-154.

10. MEDEIROS B, et al. Determinantes biopsicossociais que predizem a qualidade de vida em pessoas que vivem com HIV/AIDS. Estudos de Psicologia, 2013; 18(4): 543-550.

11. MORAES DCA, et al. Adesão de homens vivendo com HIV/Aids ao tratamento antirretroviral. Escola Anna Nery, 2014; 18(4): 676-681.

12. NASSER CAC, NEMES MIB. Representações dos participantes de uma intervenção psicossocial para melhoria da adesão ao tratamento da aids. Interface: Comunicação, Saúde e Educação, 2016; 20(58): 661-677.

13. OLIVEIRA FBM, et al. Qualidade de vida e fatores associados em pessoas vivendo com HIV/AIDS. Acta Paulista de Enfermagem, 2015; 28(6): 510-516.

14. PASCHOAL EP, et al. Adesão à terapia antirretroviral e suas representações para pessoas vivendo com HIV/Aids. Escola Anna Nery, 2014; 18(1): 32-40.

15. PONTES EVC, SANTOS ALP. Representação Social da Aids na Óptica de mulheres que vivem com HIV, PA. Trabalho de Conclusão de Curso (Graduação em Enfermagem) - Centro de Ciências Biológicas e da Saúde. Universidade do Estado do Pará, Belém, 2007.

16. RASERA EF, et al. A atuação do psicólogo em ONG/AIDS. Psicologia: Ciência e Profissão, 2007; 27(3): 566-575.

17. SILVA JB, et al. Os significados da comorbidade para os pacientes vivendo com TB/HIV: repercussões no tratamento. Physis: Revista de Saúde Coletiva, 2015; 25(1): 2009-229.

18. TAVARES NUL, et al. Fatores associados à baixa adesão ao tratamento medicamentoso em idosos. Revista de Saúde Pública, 2013; 47(6): 1092-1101. 